
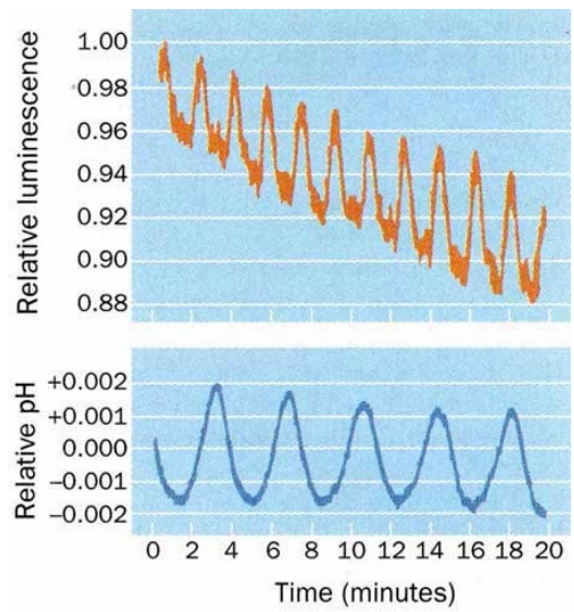

FIG. 2 When bacteriorhodopsin is incorporated into liposomes in the presence of cynanine fluorescent dyes, continuous radiation produces oscillation in the fluorescence from the dye (red trace) and in the relative $\mathrm{pH}$ (blue trace) under certain conditions. Data reproduced with permission from ref. 1 (bacteriorhodopsin concentration $2.7 \mu \mathrm{g} \mathrm{ml} \mathrm{ml}^{-1}$ actinic $(\lambda>550 \mathrm{~nm})$ intensity $55 \mathrm{~mW} \mathrm{~cm}^{-2}$. $\mathrm{pH}_{\mathrm{dark}}=6.8$ )

the observed oscillations. The first, and trivial, explanation is that the permeability of the membrane is undergoing repeated breakdown and repair, but this is unlikely given the two decades in field gradient under which oscillations were observed. The more exciting possibility is that the photoinduced proton pumping of bacteriorhodopsin includes a cooperative process involving synchronous, positive feedback.

Engineers have long recognized that frequency encoding is preferable to amplitude encoding with respect to resistance to noise and precision of control - for instance, frequency modulation (FM) is preferable to amplitude modulation (AM) for radio reception and bandwidth. Raap and co-workers have proposed that the oscillatory behaviour that characterizes many biochemical control networks is the biological analogue of the above engineering principle ${ }^{4}$. For example, some insects emit pheromones with a periodic frequency that is species-dependent. Not only does the oscillatory emission improve sensitivity for the receiver, but only members of the same species are tuned to respond to the frequency, a process known as 'odour song'.

Ross and Schell, though, suggest instead that oscillatory behaviour in biological systems is selected through evolution because it enhances thermodynamic efficiency ${ }^{5}$. The idea is that the rate of a chemical reaction is inversely proportional to the free energy barrier $(\triangle G)$ associated with the reaction step. If the barrier increases as a function of the reaction, which is often the case in biological reactions, then oscillatory behaviour can enhance efficiency by adjusting the phase between the fluxes and the forces. The dissipation is reduced if the rate is a maximum at minimum $\triangle G$ and a minimum at maximum $\triangle G$. Nonlinearities (positive feedback) in the reaction mechanism are essential for maintaining the proper phase relationships. The engineering analogue is to enhance the efficiency of an electronic device by minimizing the phase difference between voltage and current.

Regardless of origin, oscillations in biological systems are ubiquitous, and over 140 examples have been examined $^{4,5}$. All such systems can be interpreted as satisfying one of the above two models, and many appear to satisfy both. So there seems to be an evolutionary force that favours oscillatory behaviour when the available energy approaches the barrier to the process. In cases like these, nonlinear behaviour is selected to provide greater efficiency or resistance to noise.

This is essential in the archaebacterium, as the primary photochemical event of bacteriorhodopsin stores only about 12 kcal per mol (about $50 \mathrm{~kJ}$ per mol), which leaves less than $4 \mathrm{kcal}$ per mol of excess energy under nominal conditions ${ }^{6}$. The observed oscillations may represent the use of autocatalytic behaviour to optimize the efficiency. Perhaps, for instance, the protein adjusts its reverse proton permeability in response to the bulk properties of the solution so that the phase relationship between the proton pumping process and the field gradient exhibits minimal dissipation (in this case, when they are out of phase). This process is analogous to the use of a capacitor on an electric motor so that the voltage and the current are maintained in phase for greatest efficiency.

The mechanistic origins of bacteriorhodopsin's oscillatory behaviour remain to be discovered, and this unexpected autocatalytic behaviour should tell us something about the proton pumping mechanism itself. If the proton pumping process really is being optimized by a synchronous nonlinear mechanism, it only goes to show that the key optimization principles of engineering have already been implemented in biological systems.

Robert $R$. Birge is in the Department of Chemistry and the W. M. Keck Center for Molecular Electronics, Syracuse University, Syracuse, New York 13244, USA.

1. Tributsch, H. \& Bogomolni, R. A. Chem. Phys. Lett. 227 74-78 (1994)

2. Oesterhelt, D. \& Stoeckenius, W. Nature New Biol. 233 149-152 (1971)

3. Birge, R. R. Biochim. biophys. Acta 1016, 293-327 (1990)

4. Raap, P. E., Mees, A. I. \& Sparrow, C. T. J. theor. Biol, 90. $531-544(1981)$

5. Ross, J. \& Schell, M. A. Rev. Biophys. biophys. Chem. 16 , 401-422 (1987)

6. Birge, R. R. et al. J. Am. chem. Soc. 113, 4327-4328 (1991).

\section{Clearing the air}

Human bodily odours are widely feared. The soap and cosmetics industries sell many of their products not as removers of dirt, but as destroyers of smell. Daedalus points out that the volatile smelly emissions from the human frame must be very small - perhaps a few milligrams a day. This tiny outflow could easily be mopped up by the sort of absorptive charcoal found in smell. absorbing kitchen ventilators. A carbonfibre cloth is already widely used in gas masks and smoke filters; in the form of smart black underwear it should absorb body effluvia perfectly. A combined weave of carbon and polyester might be stronger and more practical.

But even the best absorption filter soon gets saturated. Carbon-based antismell underclothes could, of course, be regenerated by heating them in a vacuum; but Daedalus has a better idea. He recalls the chemical catalysts widely used to oxidize fumes and smells in industrial exhaust air. They are porous materials such as charcoal or ceramic, loaded with some finely divided catalytic metal. They usually run hotter than $100^{\circ} \mathrm{C}$, but some will work below this. DREADCO's chemists hope to perfect catalysts which will work slowly even at body heat. In an absorptive garment, they would slowly burn up entrapped odour molecules, regenerating the absorbent automatically.

Daedalus is even testing a cloth knitted from polyester and ultrafine platinum wire. This vastly expensive fabric is made for oxidizing ammonia, but it should also burn up the related amines of human effluvia. As lingerie it would provide the ultimate in costly, hygienic elegance and chic.

DREADCO's 'Catawear' should fill a long-smelt want. The fear of being unwittingly offensive to our fellows, played on so subtly by the advertisers, will be vanquished at last. Personal cleanliness will cease to obsess us, and will be downgraded to the occasional removal of annoying adherent matter. Catawear will subvert the sales, not just of deodorants, but even of soap itself.

More revolutionary still, catalytic clothes may never need washing. Volatile organic stains would be burned up, and even solid grime should slowly disappear. Each grime particle would be slowly oxidized at its points of attachment to the catalyst, whereupon it would simply fall off. Hanging in the wardrobe or bundled in the drawer, Catawear garments will slowly clean themselves. They may even scavenge grime and dead cells from the wearer's own skin, reducing sales of soap even further.

David Jones 